\title{
Laser Pulse Duration Optimization for Photothermal Therapy with Gold Nanostars
}

\author{
Juntao Cao \\ Chongqing Foreign Language School, Chongqing, China \\ Email: carl_juntaocao@outlook.com
}

How to cite this paper: Cao, J.T. (2018) Laser Pulse Duration Optimization for Photothermal Therapy with Gold Nanostars. International Journal of Medical Physics, Clinical Engineering and Radiation Oncology, 7, 391-402.

https://doi.org/10.4236/ijmpcero.2018.73032

Received: July 17, 2018

Accepted: August 21, 2018

Published: August 24, 2018

Copyright $\odot 2018$ by author and Scientific Research Publishing Inc. This work is licensed under the Creative Commons Attribution International License (CC BY 4.0).

http://creativecommons.org/licenses/by/4.0/

\begin{abstract}
Photothermal therapy (PTT), which utilizes light radiation to create localized heating effect in the targeted areas, is a promising solution for highly specific yet minimally invasive cancer therapy. PTT uses photothermal agents, which are usually nanoparticles that absorb strongly in the near-infrared optical window where minimal tissue absorption occurs. Photothermal agents are also highly functionalized to target at specific tumor sites. Gold nanostar is an ideal candidate for photothermal agents, because it not only has a Surface Plasmon Resonance in the near-infrared, but also can be easily produced and purified, and is extremely versatile in the drug delivery process. In order to achieve maximum amount of localized heating, pulse lasers are usually used in laser ablation processes like photothermal therapy. However, intensive laser radiation can cause damage to regular tissues as well the nanostructures themselves. Therefore, identifying the optimal pulse duration to effectively generate localized heating in the tumorous tissues while keeping the normal tissues and the nanostructures intact is important to achieving optimal photo-therapeutic results. This manuscript provides a numerical calculation method with Comsol Multiphysics to optimize the pulse condition of the gold nanostars under photothermal therapy settings. Based on results, gold nanostar displays significant temperature heterogeneity under femtosecond and picosecond laser radiation, while nanosecond laser only induces rather uniform heating effects across the entire gold nanostar particle. This finding indicates that femtosecond laser, which is the most common type of laser used for ablation, is likely to melt the tip of the gold nanostar before the nanostar body reaches a reasonably high temperature. Picosecond and nanosecond lasers are much less likely to induce such dramatic morphology change. This study offers important insight into finding the optimal condition for photothermal therapy with maximal efficacy and minimal damage.
\end{abstract}

\section{Keywords}

Photothermal Therapy, Photothermal Imaging, Gold Nanostars, Laser Ablation 


\section{Introduction}

Gold nanoparticles (GNP) have attracted a large amount of attention in cancer research in recent decades [1] [2] [3] [4]. When stimulated with light radiation at the proper wavelengths, the conduction electrons on the GNP surface display unique behavior of collective oscillation, which is known as the Localized Surface Plasmon Resonance (LSPR) [3] [5] [6] [7]. At the LSPR wavelengths, GNPs interact strongly with the incident light, and absorb and scatter much more efficiently than other nanoparticles of similar sizes [6] [8] [9] [10]. Another important feature of GNPs is the strong correlation between its physical shape and its optical properties. Slight differences in size, shape, geometry and the surrounding environments of GNP shift its optical spectrum dramatically. Controlling the shape and size of the GNP becomes an important handle for researchers to manipulate the optical responses. These two important features make GNP an ideal candidate for photothermal imaging [11] [12] [13] [14] and therapy [15] [16] [17] [18] [19].

In biological application settings, light therapy can be a particularly powerful tool, because it is effective, accurate, cost-effective, and induces little side effects. However, not all light interacts with the biological tissues in the same manner. Ultraviolet and visible lights can deliver a large amount of energy, but they do not penetrate deep into the tissue. For infrared light beyond $1300 \mathrm{~nm}$, it is strongly absorbed by water, which in turn restricts the light penetration depth as well. There is a range of wavelength, between 800 and $1100 \mathrm{~nm}$, where biological tissues do not absorb much light, which is known as the "biological window". An ideal therapeutic or imaging agent would be designed to interact strongly with the light in this window, because it would enable the light-nanoparticle interaction to take place deeper inside the tissue and induce fewer side effects.

Because GNPs have enhanced optical response to specific wavelength which can be manipulated through changing its physical shape and geometry, several different GNP structures have been demonstrated to be promising candidates for photo-therapy and imaging in the biological window. Gold nanorods [12] [15] [20], gold nanoshells [21] [22] and gold nanostars [16] [17] [23] [24] are examples of GNP with LSPR in the near-infrared range. Among these different gold structures, gold nanostars stand out as an ideal candidate. It can be easily synthesized, batch produced and purified [25] [26]. It also possesses different curvatures on different surfaces at the tip, on the arm and at the center of the star [27]. These unique different curvatures offer different functionalities for drug delivery purpose. Photothermal therapy with gold nanostars has been performed on animals, and promising results have been shown [16]. However, there are a few technical difficulties concerning the current medical applications of gold nanostars. One of the unique features about gold nanostars is their sharp corners. It has been well-demonstrated that there is a strong plasmon resonance field enhancement effect at the tip of gold nanostructures [28] [29]. When this strong tip enhancement effect happens in a photothermal therapy setting, it 
gives rise to the temperature heterogeneity on a single nanostar. In other words, the temperature of the tips of the gold nanostars could potentially be higher than other parts of the gold nanostar. This effect is generally a beneficial one because it gives rise to localized heating effect in a confined area. In the case of photothermal therapy of cancer, gold nanostars are delivered to tumor sites and the significantly over-heated tips of gold nanostars will disrupt the micro-environment of tumor tissues, while the normal tissues with no gold nanostars stay relatively unperturbed.

In order to achieve the maximum amount of temperature difference around gold nanostars when they are in tissue, pulse lasers are usually used because they deliver a large amount of power within a short period of time and let the normal tissue rest for an extended period of time [17] [19]. However, the energy in the laser pulses can be so high that it elevates the temperature of the GNP to above its melting point, which would cause it to melt and undergo morphology changes. Similar effects have been observed and heavily studied on gold nanorods. El-Sayed and coworkers studied the melting of gold nanorods and found out the surface defects are strongly correlated with the melting phenomenon [30]. They further determined the melting energy of gold nanorods [31]. In the case of gold nanostars, the tips are likely to reach the melting point at a lower energy density from the laser than the rest of the nanostar body. The morphology change is especially important for gold nanostars, because the sharp tips of the nanostars result in a large aspect ratio, which pins the LSPR of the gold nanostars in the near infrared range. In order to avoid this shape change, Continuous Wave (CW) lasers have been proposed for photothermal therapies. It is far safer for normal tissues and it is far less likely to melt down the gold nanostars. However, it means that we are not close to exploiting the full potential of the photothermal therapeutic effect of gold nanostars. There is a delicate balance that we need to strike here to achieve the optimal result: the laser pulses should be strong enough to significantly heat up the photothermal agents, while keeping the normal tissues and the shape of the nanostars relative unperturbed. In this manuscript, we aim to explore the optimal conditions for striking this delicate balance with our numerical calculations. We first simulate the interaction between gold nanostars and pulse laser light with different pulse duration and pulse energy levels. Then we calculate the heat transfer process when the absorbed optical power of the gold nanostars is dissipated into its surrounding environment. Temperatures of the tips of the gold nanostars and the average temperature of the structure can be obtained and compared to the melting point of gold. After a series of comparisons, we can find out the optimal pulse duration and energy level for gold nanostars in photothermal therapy settings.

\section{Material and Methods}

In this manuscript, we use Finite Element Analysis with Comsol Multiphysics 5.3 to simulate the gold nanostar interaction with light and the heat transfer 
process that follows. First of all, the shape of the gold nanostar was constructed with a center sphere fused together with six cones pointing at two opposite directions of all three axes of the Cartesian coordinates. Gold nanostars with different sizes have been used for photothermal therapy. Vo-Dinh and coworkers studied gold nanostars with $30 \mathrm{~nm}$ diameter and $60 \mathrm{~nm}$ diameter, and their therapeutic effects. Results showed that $30 \mathrm{~nm}$ gold nanostars are more effective [16]. Particles with similar sizes were used in a different study and were proven again to be robust therapeutic agents [17]. Therefore we model the gold nanostars in our study to be similar to the ones reported in literature. The diameter of the center sphere is $20 \mathrm{~nm}$, the diameters of the cones are $12 \mathrm{~nm}$ at the axes, and the height of the cones is $30 \mathrm{~nm}$. This shape matches what was reported in literature for gold nanostars used in photothermal therapy. The refractive index of gold reported by Johnson and Christy [7], and the surrounding environment is set to be water with refractive index of $1.33+0 * i$. The light absorption of water between $500 \mathrm{~nm}$ to $1300 \mathrm{~nm}$ is ignored.

First, we simulate the interaction between gold nanostar and the light in a range of wavelengths. A gold nanostar is placed at origin of the geometry and a concentric sphere encloses the gold nanostar, which represents the homogeneous aqueous environment that the nanoparticle is suspended in. A layer of Perfectly Matched Layer is wrapped around the sphere. The Electromagnetic Waves, Frequency Domain (emw) physics is selected to simulate the light-matter interaction process. The strength of the electric field is specified at different levels to simulate the different illumination intensities that the nanostar is exposed to under different laser intensities. The polarization of the light is along the $\mathrm{z}$ axis, direction of traveling is along the $\mathrm{x}$ axis. A scattering boundary is selected to be the outmost surface of the Perfectly Matched Layer. The wavelengths for calculation are from $500 \mathrm{~nm}$ to $1400 \mathrm{~nm}$, which is wider than the "optical window" that we are interested in.

Then we simulate the heat transfer process between the gold nanostar and the water environment. In order to simulate the pulsed heating process, we first calculate the absorbed power from gold nanostar under peak radiance of pulsed laser radiation. The absorbed optical power of the gold nanostars is assumed to be the heating power in the heat transfer process. Then in the "Definition" section of the Comsol Multiphysics software, we define an analytical function, which denotes a Gaussian distribution of power over the period of pulse duration, with the highest value of intensity as unity. We then select the Heat Transfer in Fluids (ht) physics and define a heat source as the gold nanostar. The power of the heat source is defined to be the absorbed optical power from the light-particle calculation multiplied by the pulse function that we defined earlier. The heat equation in the heat transfer process is:

$$
\begin{gathered}
\rho C_{p} \frac{\partial T}{\partial t}+\rho C_{p} u \cdot \nabla T+\nabla \cdot q=Q \\
q=-k \nabla \mathrm{T}
\end{gathered}
$$


where $\rho$ is the fluid density; $C_{p}$ is the fluid heat capacity at given pressure; $k$ is the fluid thermal conductivity; $u$ is the fluid velocity field; $Q$ is the heat source. An important advantage of Comsol Multiphysics pertaining to our study is that it is able to couple the absorbed optical power from the Electromagnetic Waves (emw) calculation directly in to the Heat Transfer in Fluid (ht) study in every finite element in three dimensions. In other words, with Comsol Multiphysics, we are able to readily map out the different heating power from different parts of the gold nanostars and their resulting different temperatures. The gold nanostars are modeled as pulsed heating sources with a Gaussian pulse shape and pulse durations of 100 femtoseconds, 100 picoseconds, and 100 nanoseconds respectively. It reaches the maximum heating power, as the absorbed power from the Electromagnetic Waves study (emw.Qh), at the center of the pulse. The repetition rate of the laser is set to be $10 \mathrm{~Hz}$, which is common for lasers for phototherapies.

It is worth noting that this treatment is a little different from the realistic physical process. In reality, when the nanostar interacts with the pulse laser, the nanostar interacts with the light under different intensities in the pulsing period of the laser, which in turn translates to different heating powers. However, since the absorbed power from nanostar is proportional to the illumination power, and the simulation of light-matter interaction over time is much more difficult because it involves calculations of both frequency-domain and time-domain at the same time, we simply simulate the light-matter interaction under peak radiance and obtained the peak heating power and treated the gold nanostar as a pulsed heating source in the time domain in the heat transfer study. Our treatment does not make any approximation and is effectively equivalent to the physical process that we are interested. This treatment saves a large amount of computational power without making any compromises.

\section{Results and Discussion}

In Figure 1(b), we can clearly see that this gold nanostar structure has a strong LSPR peak in the near infrared range as we desired. The absorption peak is around $830 \mathrm{~nm}$. In Figure 2, the electric field distribution on and near the gold nanostar are shown for (a) femtosecond, (b) picosecond and (c)nanosecond pulses. It is clearly shown that the electric field is much stronger at the tips of the gold nanostars than other parts no matter what the pulse duration is. This observation agrees with what has been reported before, known as the tip enhancement effect [16] [23] [28]. The degree of heterogeneity is larger for (a) femtosecond laser, compared to (b) picosecond and (c) nanosecond, because the peak radiance of the incident light is far more intense in the case of femtosecond laser.

As a result of the heterogeneity of electric field in space, the temperature profile under the peak radiance intensity is also different at the tips. However, as shown in Figures 2(d)-(f), temperatures at the nanostar tips reaches a significantly higher level under femtosecond laser radiation, while this heterogeneity in 
(a)

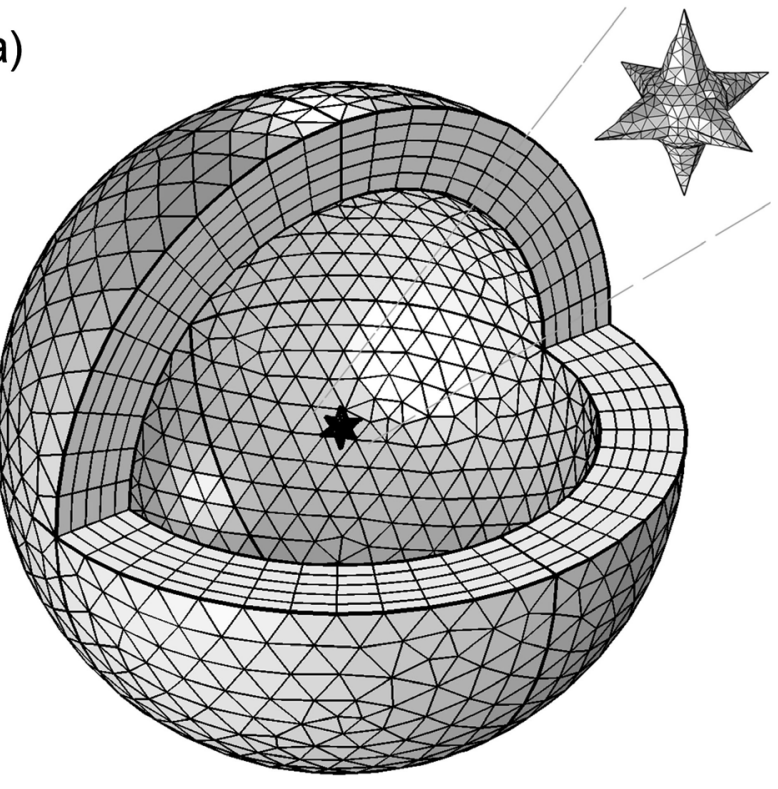

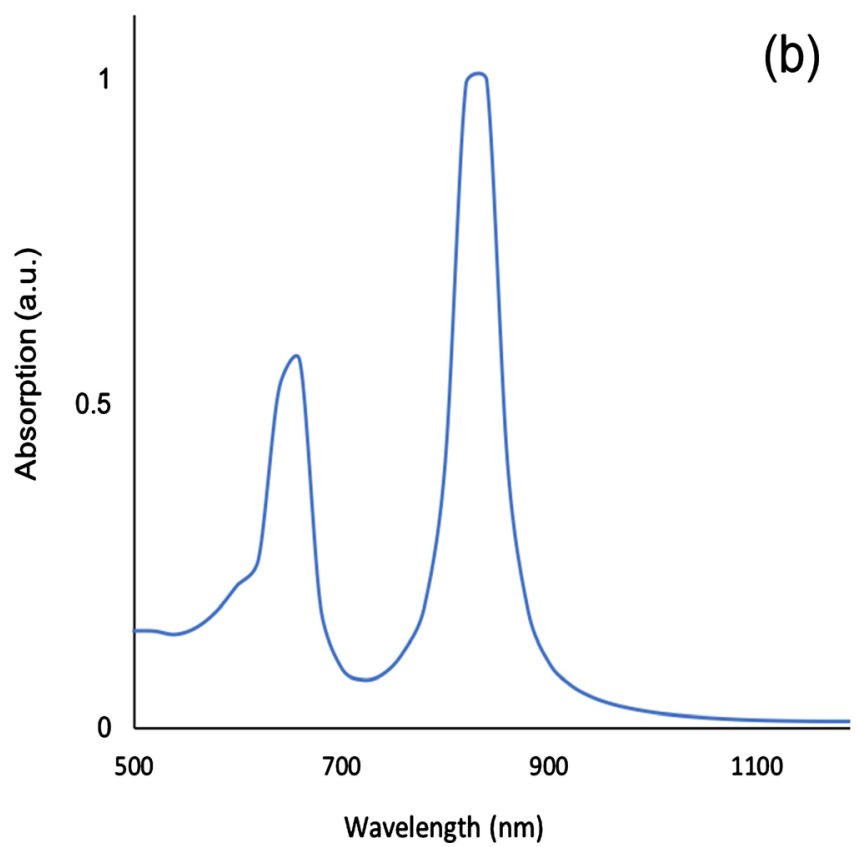

Figure 1. (a) Geometry and meshing of the simulated model; (b) Simulated absorption spectrum of gold nanostar in water.

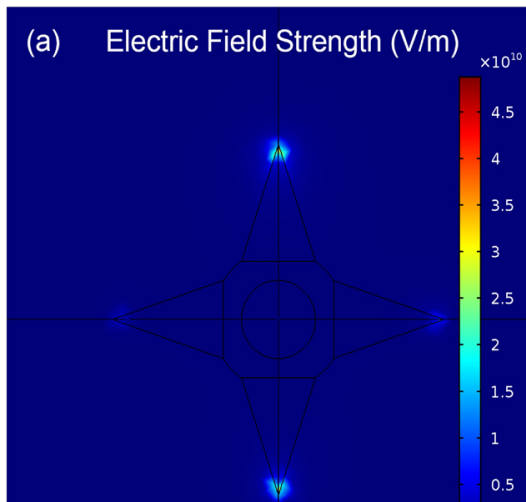

Femtosecond Pulse

(d) Temperature Difference Profile (K)

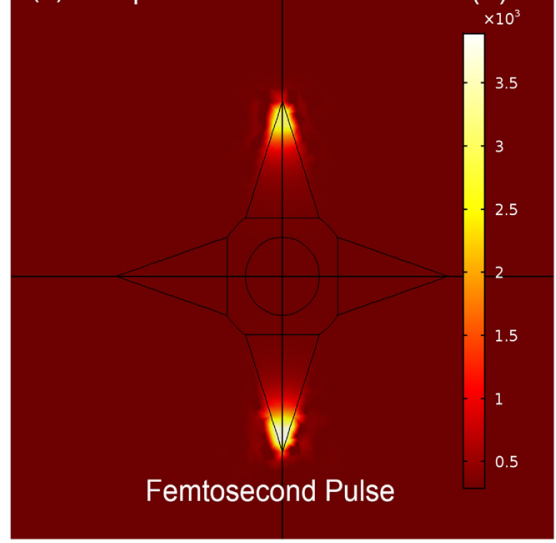

(b) Electrical Field Strength (V/M)

A

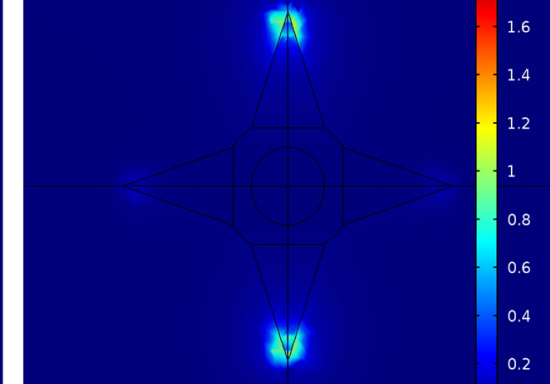

Picosecond Pulse

(e) Temperature Difference Profile (K)

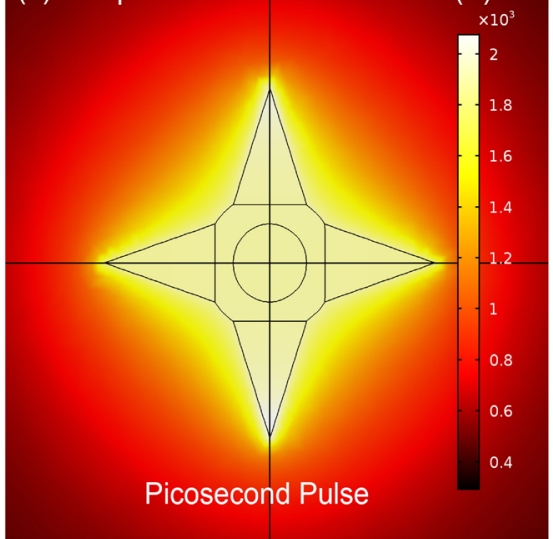

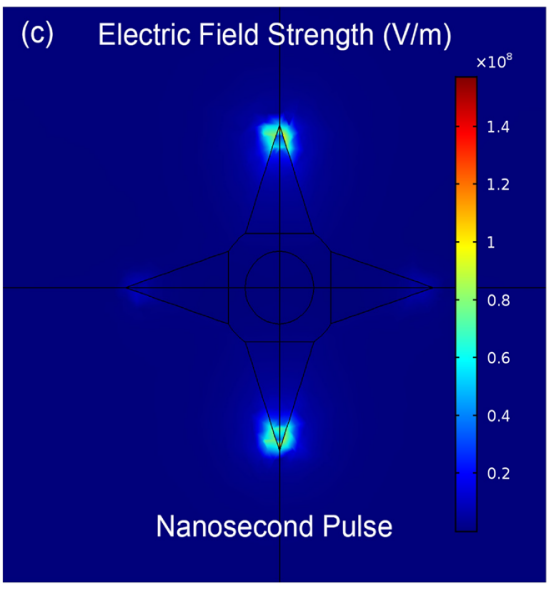

(f) Temperature Difference Profile (K)

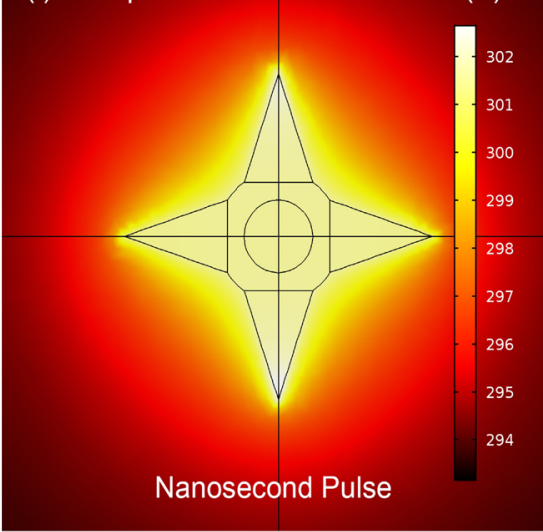

Figure 2. (a)-(c) are the distribution of electric field in space for femtosecond, picosecond and nanosecond pulses respectively; (d)-(f) are the temperature distribution in space at the peak temperature of gold nanostar for femtosecond, picosecond and nanosecond pulses respectively. 
temperature is not visible in the cases of picosecond laser and nanosecond lasers. When paring the electric field distribution graphs (a)-(c), together with the corresponding temperature profile graphs of (d)-(f), it is clear that the distribution heterogeneity in electric field translates to temperature heterogeneity in case of femtosecond laser radiation, but not in the cases of picosecond and nanosecond lasers. This interesting observation can be explained by the heat transfer process.

As we know, the temperature of an object is determined by the amount of heat that this object takes in, the amount of heat that the object gives out and the heat capacity of the object. When the gold nanostar interacts with the electromagnetic field, the electric field is going to be much more intense at the tip of the gold nanostar, because the electric field on the surface of gold nanostar is determined by the collective oscillation of the conduction electrons on the surface, also known as the Surface Plasmon Enhancement (SPR) effect. The surface to volume ratio at the tip of the nanostar is far larger than anywhere else on the body. Therefore the electrons are far more crowded at the tips, which resulted in tip enhancement effect. However, because the surface to volume ratio is high at the tips, it is much easier for the tip to cool down through convective heat transfer with a given amount of heat input. Therefore although it is true that the electric field is always going to be much more intense at the tips, which will result in much more intense heat generation, no matter what pulse duration is, cooling is also much quicker at the tip as well. This competing effect manifests itself clearly in Figure 3.

Tables 1-3 show the highest temperatures that the tips and the body of the gold nanostar reach under different peak radiance and pulse durations. In Table 1 , under 100 femtosecond pulse radiation, the difference of the temperature rises between the tip and the body of the gold nanostar is very large. For example, under $663.1 \mathrm{TW} / \mathrm{m}^{2}$ peak radiance, the tip temperature rise can reach as high as $46321 \mathrm{~K}$, while the average temperature of the nanostar body only rises by 1814 K. In Table 2, under 100 picosecond pulse radiation, such difference is still clearly present, but is far less dramatic. In Table 3, under 100 nanosecond pulse
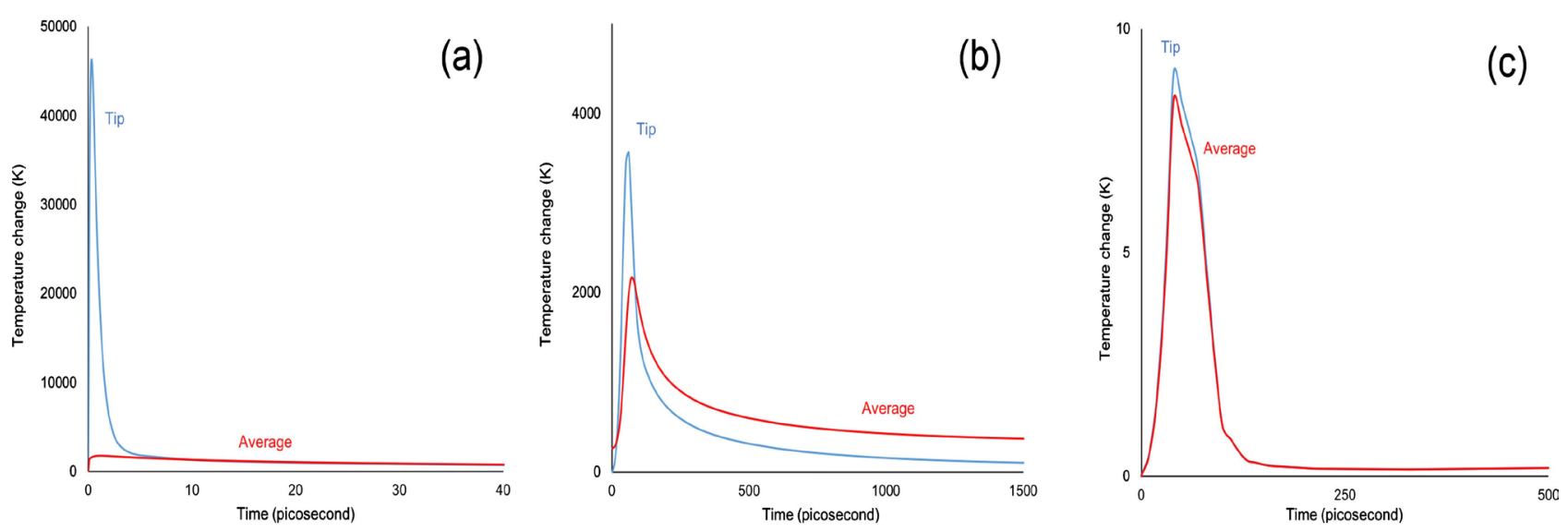

Figure 3. (a)-(c) are exemplary temperature decay curves of gold nanostar in water after heating by femtosecond, picosecond and nanosecond laser pulses. 
Table 1. Temperature rise of the tip and the nanostar body under different peak radiance with 100 femtosecond pulse duration. The number marked in yellow are above the temperature rise need to melt gold.

\begin{tabular}{ccc}
\hline Peak Radiance $\left(10^{12} \mathrm{~W} / \mathrm{m}^{2}\right)$ & $\begin{array}{c}\text { Peak Temperature Change } \\
\text { of the Tip }(\mathrm{K})\end{array}$ & $\begin{array}{c}\text { Peak Temperature Change } \\
\text { of the Gold Nanostar Overall }(\mathrm{K})\end{array}$ \\
\hline 9.5 & 25 & 1 \\
17.0 & 1249 & 51 \\
26.5 & 1603 & 65 \\
106.1 & 7769 & 302 \\
238.7 & 17633 & 688 \\
663.1 & 46321 & 1814 \\
\hline
\end{tabular}

Table 2. Temperature rise of the tip and the nanostar body under different peak radiance with 100 picosecond pulse duration. The number marked in yellow are above the temperature rise need to melt gold.

\begin{tabular}{ccc}
\hline Peak Radiance $\left(10^{9} \mathrm{~W} / \mathrm{m}^{2}\right)$ & $\begin{array}{c}\text { Peak Temperature Change } \\
\text { of the Tip }(\mathrm{K})\end{array}$ & $\begin{array}{c}\text { Peak Temperature Change } \\
\text { of the Gold Nanostar Overall (K) }\end{array}$ \\
\hline 95.5 & 241 & 119 \\
265.3 & 716 & 373 \\
596.8 & 1626 & 847 \\
1061.0 & 2874 & 1504 \\
1283.8 & 3566 & 1901 \\
\hline
\end{tabular}

Table 3. Temperature rise of the tip and the nanostar body under different peak radiance with 100 nanosecond pulse duration. The number marked in yellow are above the temperature rise need to melt gold.

\begin{tabular}{ccc}
\hline Peak Radiance $\left(10^{6} \mathrm{~W} / \mathrm{m}^{2}\right)$ & $\begin{array}{c}\text { Peak Temperature Change } \\
\text { of the Tip }(\mathrm{K})\end{array}$ & $\begin{array}{c}\text { Peak Temperature Change } \\
\text { of the Gold Nanostar Overall }(\mathrm{K})\end{array}$ \\
\hline 0.7 & 9 & 8 \\
2.7 & 44 & 41 \\
23.9 & 402 & 377 \\
95.5 & 1702 & 1602 \\
\hline
\end{tabular}

radiation, the temperature rises of the tip of the gold nanostar and the average body almost the same. This comparison is also shown in Figure 3 with the time decay curves.

Figure 4 displays the results from Tables 1-3 and compare them to the melting temperature of gold. In Figure 4(a), it is clearly shown that femtosecond pulses will melt the tips much earlier than it melt the body of the nanostars. Figure 4(b) shows that with picosecond pulses, the tips will still melt significantly earlier than the nanostar body, but it is possible to heat the gold nanostar body up to a relatively high temperature before melting its tips. Figure 4(c) shows that nanosecond pulses do not induce much temperature heterogeneity 

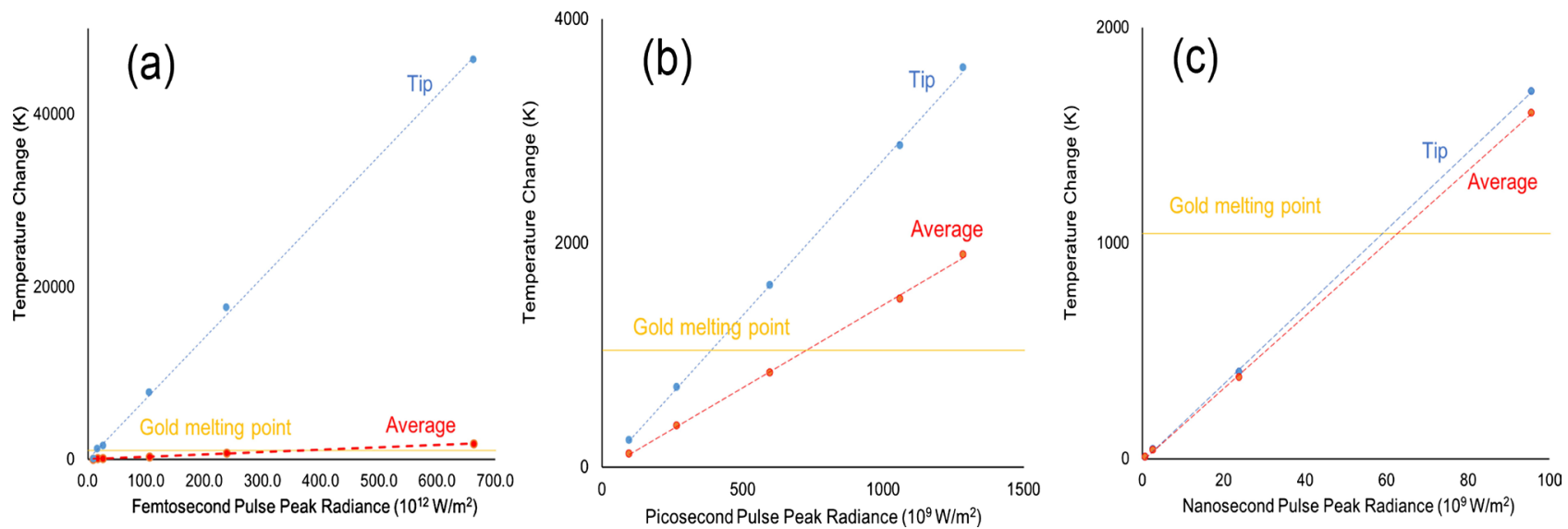

Figure 4. (a)-(c) are the peak temperatures of the tip of the gold nanostar versus the average temperature of the entire gold nanostar at different laser peak radiance levels for femtosecond, picosecond and nanosecond pulses. The melting point of gold is specified in the graphs for comparison.

on the gold nanostar. It resembles the behavior that one would expect from a Continuous Wave laser. Therefore, there is no need to widen the pulse duration further down.

Based on these tables and graphs, we can clearly observe that under femtosecond laser pulses, the temperature difference between the tips of the gold nanostars and the gold nanostar body is very significant. Such difference is also present under picosecond pulse radiation, but is very minimal under nanosecond laser pulses. This important finding indicates that femtosecond lasers, which are very popular in laser ablation applications, might not be the best option for photothermal therapy with gold nanostars. Picosecond lasers, on the other hand, can generate enough localized heating effect without easily melting the tips of the gold nanostars. Nanosecond lasers behave very similarly to Continuous Wave laser, which does not induce too much temperature heterogeneity on gold nanostars.

\section{Conclusion}

In this manuscript, the temperature change of gold nanostars under pulse laser with different durations is studied. The temperature heterogeneity caused by the tip enhancement effect of gold nanostar is carefully investigated with different peak radiance and pulse durations. Based on the results, femtosecond laser heats up the tips of the gold nanostars to a much higher $(\sim 20 \times)$ temperature rise compared to the gold nanostar body. Picosecond laser creates similar temperature heterogeneity, but the difference is not nearly as dramatic $(\sim 2 \times)$. Nanosecond laser does not induce much temperature heterogeneity. Therefore, femtosecond laser, which is one of the most popular choices for phototherapy, might not be suitable for photothermal therapy with gold nanostars. Because it is likely to melt the tips of the nanostar, which in turn changes the absorption spectrum entirely, before the body of the nanostar reaches a reasonably high temperature. Picosecond second lasers, on the other hand, induce a manageable temperature 
heterogeneity which can be an ideal option for this application. Nanosecond lasers behave very similarly to CW lasers in this case, which might not be a good candidate because of the elevated costs compared to CW lasers. In the future, experiments will be conducted to validate this simulation. This study offers important insights into selecting the optimal laser for photothermal therapy, which can potentially be a highly specific and minimally invasive tool for cancer therapy.

\section{Conflicts of Interest}

The authors declare no conflicts of interest regarding the publication of this paper.

\section{References}

[1] Jain, P.K., Lee, K.S., El-Sayed, I.H. and El-Sayed, M.A. (2006) Calculated Absorption and Scattering Properties of Gold Nanoparticles of Different Size, Shape, and Composition: Applications in Biological Imaging and Biomedicine. The Journal of Physical Chemistry B, 110, 7238-7248. https://doi.org/10.1021/jp057170o

[2] Eustis, S. and El-Sayed, M.A. (2006) Why Gold Nanoparticles Are More Precious than Pretty Gold: Noble Metal Surface Plasmon Resonance and Its Enhancement of the Radiative and Nonradiative Properties of Nanocrystals of Different Shapes. Chemical Society Reviews, 35, 209-217. https://doi.org/10.1039/B514191E

[3] Hutter, E. and Fendler, J. H. (2004) Exploitation of Localized Surface Plasmon Resonance. Advanced Materials, 16, 1685-1706. https://doi.org/10.1002/adma.200400271

[4] Li, Z., Mao, W., Devadas, M.S. and Hartland, G.V. (2015) Absorption Spectroscopy of Single Optically Trapped Gold Nanorods. Nano Letters, 15, 7731-7735. https://doi.org/10.1021/acs.nanolett.5b03833

[5] Johns, P., Yu, K., Devadas, M.S., Li, Z., Major, T.A. and Hartland, G.V. (2014) Effect of Substrate Discontinuities on the Propagating Surface Plasmon Polariton Modes in Gold Nanobars. Nanoscale, 6, 14289-14296. https://doi.org/10.1039/C4NR04131C

[6] Del Fatti, N., Christofilos, D., Vallée, F. and Christofilos, D. (2008) Optical Response of a Single Gold Nanoparticle. Gold Bulletin, 41, 147-158. https://doi.org/10.1007/BF03216592

[7] Johnson, P.B. and Christy, R.W. (1972) Optical Constants of the Noble Metals. Physical Review B, 6, 4370-4379. https://doi.org/10.1103/PhysRevB.6.4370

[8] Devadas, M.S., Li, Z., Major, T.A., Lo, S.S., Havard, N., Yu, K., Johns, P. and Hartland, G.V. (2013) Detection of Single Gold Nanoparticles Using Spatial Modulation Spectroscopy Implemented with a Galvo-Scanning Mirror System. Applied Optics, 52, 7806-7811. https://doi.org/10.1364/AO.52.007806

[9] Devadas, M.S., Li, Z. and Hartland, G.V. (2014) Imaging and Analysis of Single Optically Trapped Gold Nanoparticles Using Spatial Modulation Spectroscopy. The Journal of Physical Chemistry Letters, 5, 2910-2915. https://doi.org/10.1021/jz501409q

[10] Devadas, M.S., Devkota, T., Johns, P., Li, Z., Lo, S.S., Yu, K., Huang, L. and Hartland, G.V. (2015) Imaging Nano-Objects by Linear and Nonlinear Optical Absorption Microscopies. Nanotechnology, 26, Article ID: 354001. 
https://doi.org/10.1088/0957-4484/26/35/354001

[11] Li, Z., Aleshire, K., Kuno, M. and Hartland, G.V. (2017) Super-Resolution Far-Field Infrared Imaging by Photothermal Heterodyne Imaging. The Journal of Physical Chemistry B, 121, 8838-8846. https://doi.org/10.1021/acs.jpcb.7b06065

[12] Ling, T., Qingshan, W., Alexander, W. and Cheng, J. (2009) Gold Nanorods as Contrast Agents for Biological Imaging: Optical Properties, Surface Conjugation and Photothermal Effects. Photochemistry and Photobiology, 85, 21-32. https://doi.org/10.1111/j.1751-1097.2008.00507.x

[13] Kim, J.-W., Galanzha, E.I., Shashkov, E.V., Moon, H.M. and Zharov, V.P. (2009) Golden Carbon Nanotubes as Multimodal Photoacoustic and Photothermal High-Contrast Molecular Agents. Nature Nanotechnology, 4, 688. https://doi.org/10.1038/nnano.2009.231

[14] Li, Z., Kuno, M. and Hartland, G.V. (2015) Super-Resolution Imaging with Mid-IR Photothermal Microscopy on the Single Particle Level. SPIE Proceedings of Physical Chemistry of Interfaces and Nanomaterials XIV, 9549, Article ID: 954912. https://doi.org/10.1117/12.2188337

[15] Huang, X., El-Sayed, I.H., Qian, W. and El-Sayed, M.A. (2006) Cancer Cell Imaging and Photothermal Therapy in the Near-Infrared Region by Using Gold Nanorods. Journal of the American Chemical Society, 128, 2115-2120. https://doi.org/10.1021/ja057254a

[16] Liu, Y., Ashton, J.R., Moding, E.J., Yuan, H., Register, J.K., Fales, A.M., Choi, J., Whitley, M.J., Zhao, X., Qi, Y., Ma, Y., Vaidyanathan, G., Zalutsky, M.R., Kirsch, D.G., Badea, C.T. and Vo-Dinh, T. (2015) A Plasmonic Gold Nanostar Theranostic Probe for In Vivo Tumor Imaging and Photothermal Therapy. Theranostics, 5, 946-960. https://doi.org/10.7150/thno.11974

[17] Yuan, H., Fales, A.M. and Vo-Dinh, T. (2012) TAT Peptide-Functionalized Gold Nanostars: Enhanced Intracellular Delivery and Efficient NIR Photothermal Therapy Using Ultralow Irradiance. Journal of the American Chemical Society, 134, 11358-11361. https://doi.org/10.1021/ja304180y

[18] Huang, X., Jain, P.K., El-Sayed, I.H. and El-Sayed, M.A. (2007) Plasmonic Photothermal Therapy (PPTT) Using Gold Nanoparticles. Lasers in Medical Science, 23, 217-228. https://doi.org/10.1007/s10103-007-0470-x

[19] Pustovalov1, V.K., Smetannikov, A.S. and Zharov, V.P. (2008) Photothermal and Accompanied Phenomena of Selective Nanophotothermolysis with Gold Nanoparticles and Laser Pulses. Laser Physics Letters, 5, 775-792. https://doi.org/10.1002/lapl.200810072

[20] Xiaohua, H., Svetlana, N. and El-Sayed, M.A. (2009) Gold Nanorods: From Synthesis and Properties to Biological and Biomedical Applications. Advanced Materials, 21, 4880-4910. https://doi.org/10.1002/adma.200802789

[21] Oldenburg, S.J., Jackson, J.B., Westcott, S.L. and Halas, N.J. (1999) Infrared Extinction Properties of Gold Nanoshells. Applied Physics Letters, 75, 2897-2899. https://doi.org/10.1063/1.125183

[22] Prevo, B.G., Esakoff, S.A., Mikhailovsky, A. and Zasadzinski, J.A. (2008) Scalable Routes to Gold Nanoshells with Tunable Sizes and Their Response to Near Infrared Pulsed Laser Irradiation. Small, 4, 1183-1195. https://doi.org/10.1002/smll.200701290

[23] Chatterjee, H., Rahman, D.S., Sengupta, M. and Ghosh, S.K. (2018) Gold Nanostars in Plasmonic Photothermal Therapy: The Role of Tip Heads in the Thermoplasmonic Landscape. The Journal of Physical Chemistry C, 122, 13082-13094. 
https://doi.org/10.1021/acs.jpcc.8b00388

[24] Dam, D.H.M., Culver, K.S.B. and Odom, T.W. (2014) Grafting Aptamers onto Gold Nanostars Increases in Vitro Efficacy in a Wide Range of Cancer Cell Types. Molecular Pharmaceutics, 11, 580-587. https://doi.org/10.1021/mp4005657

[25] Kumar, P.S., Pastoriza-Santos, I., Rodríguez-González, B., Javier García de Abajo, F. and Liz-Marzán, L.M. (2008) High-Yield Synthesis and Optical Response of Gold Nanostars. Nanotechnology, 19, Article ID: 015606. https://doi.org/10.1088/0957-4484/19/01/015606

[26] Nehl, C.L., Liao, H. and Hafner, J.H. (2006) Optical Properties of Star-Shaped Gold Nanoparticles. Nano Letters, 6, 683-688. https://doi.org/10.1021/nl052409y

[27] Rotz, M.W., Culver, K.S., Parigi, G., MacRenaris, K.W., Luchinat, C., Odom, T.W. and Meade, T.J. (2015) High Relaxivity Gd(III)-DNA Gold Nanostars: Investigation of Shape Effects on Proton Relaxation. ACS Nano, 9, 3385-3396. https://doi.org/10.1021/nn5070953

[28] Sundaramurthy, A., Crozier, K.B., Kino, G.S., Fromm, D.P., Schuck, P.J. and Moerner, W.E. (2005) Field Enhancement and Gap-Dependent Resonance in a System of Two Opposing Tip-to-Tip Au Nanotriangles. Physical Review B, 72, Article ID: 165409. https://doi.org/10.1103/PhysRevB.72.165409

[29] Schuck, P.J., Fromm, D.P., Sundaramurthy, A., Kino, G.S. and Moerner, W.E. (2005) Improving the Mismatch between Light and Nanoscale Objects with Gold Bowtie Nanoantennas. Physical Review Letters, 94, Article ID: 017402. https://doi.org/10.1103/PhysRevLett.94.017402

[30] Link, S., Wang, Z.L. and El-Sayed, M.A. (2000) How Does a Gold Nanorod Melt? The Journal of Physical Chemistry B, 104, 7867-7870. https://doi.org/10.1021/jp0011701

[31] Link, S. and El-Sayed, M.A. (2001) Spectroscopic Determination of the Melting Energy of a Gold Nanorod. The Journal of Chemical Physics, 114, 2362-2368. https://doi.org/10.1063/1.1336140 